\title{
Introducción de rúbricas en el plan de Gestión de la Calidad, aplicado durante la redacción de proyectos industriales, como parte del proceso de mejora continua \\ Introduction of rubrics in the Quality Management plan, applied during the drawing-up of industrial projects, as part of the process of continuous improvement
}

\author{
Ana Cristina Royo-Sánchez, Pedro Ubieto-Artur, Ramón Miralbes-Buil \\ crisroyo@unizar.es, pubieto@unizar.es,miralbes@unizar.es \\ Departamento de Ingeniería de Diseño y Fabricación \\ Universidad de Zaragoza \\ Zaragoza, España
}

\begin{abstract}
Resumen- El objetivo de este trabajo es presentar la experiencia de mejora llevada a cabo en la asignatura de Oficina Técnica del Grado en Ingeniería en Diseño Industrial y Desarrollo de Producto de la Universidad de Zaragoza. Con esta mejora se quiere conseguir que los alumnos sean capaces de evaluar los proyectos que tienen que redactar, para así, asegurar la calidad de los documentos que los componen dentro de un ciclo de mejora continua. La mejora introducida consiste en relacionar las métricas y listas de comprobación elaboradas por los alumnos, como parte del plan de Gestión de la Calidad que aplicaran durante la redacción del proyecto, con las rúbricas empleadas por los profesores para la corrección de los trabajos. Una de las principales conclusiones que se pueden extraer de dicha experiencia es que el aplicar la mejora presenta un efecto significativo en la mejora de las calificaciones y la reducción del número de apartados con errores repetidos.
\end{abstract}

Palabras clave: Plan de Gestión de la Calidad del Proyecto, Listas de comprobación, Rúbricas, Mejora continua

Abstract- The goal of this paper is to present the improvement experience carried out in the subject of Oficina Técnica of the Degree in Engineering in Industrial Design and Product Development of Zaragoza University. With this improvement we want to get students to be able to evaluate the projects they have to write, to ensure the quality of the documents that compose those project within a cycle of continuous improvement. Students develop metrics and checklists as part of the Quality Management plan that they will apply during the drawing-up of the project. The improvement introduced is to relate these metrics and checklists with the rubrics used by the teachers for the correction of the works. One of the main conclusions that can be drawn from this experience is that applying the improvement has a significant effect on improving grades and reducing the number of sections with repeated errors.

Keywords: Project Quality Management Plan, Checklists, Rubrics, Continuous Improvement

\section{INTRODUCCIÓN}

En el nuevo contexto educativo, debido a la adaptación al Espacio Europeo de Educación Superior (EEES), la educación debe estar centrada en el aprendizaje del alumno (de Miguel, 2005; Rodríguez, 2007) y "más concretamente en los resultados de aprendizaje expresados en términos de competencias" (Cano, 2008). Además, para conseguir un sistema educativo de calidad es necesario que el profesorado cambie el proceso evaluador (Cano, 2008; Menéndez, 2014) entendiéndolo como un proceso de mejora continua y que el aprendizaje así obtenido, sea permanente (Granados, 2013).

Unos de los instrumentos que más se utilizan en la evaluación de competencias son las rúbricas (Carrizosa, 2011; Menéndez, 2014) y las listas de comprobación (Cano, 2008). En múltiples artículos se definen las rúbricas (Andrade, 2005; Fernandez, 2010; Gatica \& Uribarren, 2013; Mertler, 2001) y se indican las ventajas y desventajas de su uso para alumnos y profesores (Goodrich, 2000; Martínez, 2008). Entre las ventajas destaca la retroalimentación que obtiene el alumno de su propio aprendizaje y el profesor sobre la eficacia de los métodos empleados. Estas ventajas se incrementan con las llamadas e-rúbricas ("rúbricas construidas con distintos programas informáticos residentes o disponibles en la red" definición de (Pickett \& Dodge, 2007)) ya que la retroalimentación obtenida es mucho más rápida e inmediata (Cebrián \& Monedero, 2009; Gatica \& Uribarren, 2013).

En el caso de la asignatura de Oficina Técnica del Grado en Ingeniería en Diseño Industrial y Desarrollo de Producto de la Universidad de Zaragoza, uno de los resultados de aprendizaje que el alumno debe demostrar es: "adquieren la capacidad de desarrollar la actividad profesional dentro del ámbito del diseño industrial en lo que se refiere a interpretar y desarrollar la documentación del proyecto, así como la documentación técnica relacionada” (Universidad de Zaragoza, 2016). Otro resultado de aprendizaje buscado es el de: "realiza y lleva a cabo la planificación, programación, control y el seguimiento de un proyecto en la Oficina Técnica” (Universidad de Zaragoza, 2016), tareas éstas responsabilidad de la dirección de proyectos. 
Según Project Management Institute (PMI) el éxito de la dirección de proyectos se mide, entre otras cosas, por la calidad del proyecto, por lo que una de las Áreas de Conocimiento en las que se agrupan los 47 procesos de la dirección de proyectos identificados en la Guía de los Fundamentos para la dirección de Proyectos (Guía del PMBOK) es la Gestión de la Calidad de los Proyectos (Project Mangement Institute, 2013). Estos procesos se categorizan en cinco Grupos de Procesos (Inicio, Planificación, Ejecución, Monitoreo y Control y Cierre) que se corresponden con el proceso de mejora continua.

Para asegurar la calidad de sus trabajos, los alumnos de la asignatura de Oficina Técnica tienen que elaborar e implementar un plan de Gestión de la Calidad aplicado durante la redacción del proyecto, tomando como referencia la norma UNE 157001:2014.

El objetivo de este trabajo consiste en lograr que el alumno sea capaz de evaluar su trabajo, para asegurar la calidad de los documentos de sus proyectos dentro de un ciclo de mejora continua. La mejora propuesta en este trabajo consiste en relacionar las métricas y listas de comprobación elaboradas por los alumnos, como parte del plan de Gestión de la Calidad, con las rúbricas empleadas por los profesores para la corrección de los trabajos. De esta manera, las correcciones realizadas una vez se ha finalizado el proyecto, permiten a los alumnos por un lado, completar las listas de comprobación del plan de Gestión de la Calidad y, por otro, volver a analizar los ítems de la lista de comprobación dados por verificados por parte de los alumnos pero corregidos como erróneos por los profesores.

\section{CONTEXTO}

\section{A. Contexto y público objetivo}

La experiencia se ha realizado con los alumnos del cuarto curso (66 alumnos en el curso 2016-2017) del Grado en Ingeniería en Diseño Industrial y Desarrollo de Producto que cursan la asignatura de Oficina Técnica (obligatoria de 6.0 créditos ECTS) impartida durante el primer semestre.

Las actividades de evaluación de esta asignatura constan de un examen teórico y trabajo práctico. Dicho trabajo consiste en la realización de dos proyectos de ejecución, según la norma UNE 157001:2014 realizados en grupos de trabajo de 3 personas cada uno. El primero representa el 25\% de la nota final y parte de los diseños realizados en la asignatura de Taller de Diseño III (tercer curso de la titulación). El segundo trabajo representa el $50 \%$ de la nota final y se realiza a partir de los diseños realizados en la asignatura de Metodología del Diseño. De cada uno de los proyectos se evalúa: la dirección y gestión del trabajo realizado (un 33\%), la información aportada por los planos (un 33\%) y el formato y contenido del resto de los documentos del proyecto (el 34\%). Es en este último apartado en el que se centra la mejora propuesta.

Los alumnos dirigen, gestionan y redactan estos dos proyectos sobre un sistema de Gestión de Oficinas Técnicas. Este Sistema consiste en un entorno de trabajo de la Web 2.0 y se viene desarrollando por parte de los profesores de la asignatura desde el curso académico 2012-2013. Dentro de este sistema de Gestión se incluye el módulo de corrección por rúbricas utilizado por el equipo docente, de forma que quedan enlazados los apartados del proyecto con las correcciones planteadas.

\section{B. Necesidad}

Los grupos de trabajo elaboran el plan de Gestión de la Calidad a seguir durante la redacción del proyecto como parte de su plan de Dirección del Proyecto (Project Mangement Institute, 2013). A lo largo de los últimos cursos, se ha comprobado que su implementación no resulta satisfactoria ya que se detectan muchos errores en los documentos entregados. Algunos errores son debidos a una interpretación parcial o incorrecta de los requisitos establecidos por la norma, pero la mayor parte de los errores son debidos a falta de atención durante el proceso de redacción y de autocorrección del proyecto. Teniendo en cuenta que estos errores afectan al 34\% de la calificación de cada proyecto, se ha planteado actuar sobre el plan de Gestión de la Calidad confeccionado por los alumnos, para que sean capaces de asegurar de forma óptima la calidad de los trabajos entregados. Aprovechando que cada grupo de trabajo realiza dos proyectos, se ha visto la necesidad de añadir el resultado de la corrección del primer proyecto al ciclo de mejora continua en el que están inmersos.

\section{DESCRIPCIÓN}

Los grupos de trabajo utilizan el sistema de Gestión de Oficinas Técnicas. A grandes rasgos, este Sistema les permite redactar el proyecto, dirigir y gestionar el trabajo, enlazar los planos con el sistema de Diseño Asistido por Ordenador, además de otras utilidades. Dentro de dicho Sistema también está incluido el módulo de Gestión de la Calidad con el que los alumnos pueden, entre otras cosas, añadir listas de comprobación, vincular estas listas a las distintas tareas planificadas en el trabajo y enlazar las listas con los proyectos para firmar su verificación.

Los criterios propuestos a los alumnos para la elaboración de las listas de comprobación son los siguientes:

- Se deben definir una serie de ítems a comprobar por cada uno de los apartados que componen el proyecto, siguiendo los criterios establecidos por la norma UNE 157001.

- La métrica aplicada únicamente consistirá en indicar si se verifica lo especificado en la norma o no.

Así, por ejemplo, el apartado "1.5 Definiciones y abreviaturas” del documento Memoria del proyecto, indica que "En este capítulo de la memoria se deben relacionar todas las definiciones, abreviaturas, etc. que se han utilizado y su significado”(AENOR, 2014). Para comprobar que el apartado es correcto, las comprobaciones a realizar por parte del alumno serían:

- $\quad$ En el proyecto no se encuentran términos que necesiten una explicación o en este apartado se incluyen las definiciones de los términos que lo necesitan (Verificado / NO verificado).

- En el proyecto no se utilizan abreviaturas o en este apartado se incluyen las abreviaturas empleadas en el proyecto (Verificado / NO verificado).

Las rúbricas de corrección utilizadas por el equipo docente serían, en este caso: 
- $\quad$ En el proyecto se encuentran términos que precisan una explicación y no aparece su definición en este apartado.

- $\quad$ En el proyecto se utilizan abreviaturas y no aparecen en este apartado.

\section{A. Metodología}

Antes del comienzo de las clases presenciales, el equipo docente elabora y publica el material de referencia en la plataforma Moodle de la Universidad de Zaragoza.

El primer día de clase, el equipo docente informa a los alumnos de que deben realizar dos proyectos de ejecución, con su correspondiente plan de Dirección (Project Mangement Institute, 2013) en grupos de trabajo de 3 personas. Para llevar a cabo estos trabajos utilizarán el sistema de Gestión de Oficinas Técnicas desarrollado.

El equipo docente explica el funcionamiento del sistema de Gestión mediante actividades presenciales (clases magistrales de teoría y problemas además de prácticas de laboratorio) durante las primeras semanas del curso.

Los grupos de trabajo desarrollan los dos proyectos en el sistema de Gestión. Este Sistema permite al equipo docente la monitorización de todos los trabajos en curso, lo que facilita su seguimiento y la resolución de dudas en tiempo real.

Durante las fases iniciales del primer proyecto, cada grupo elabora, entre otros documentos, el plan de Gestión de Calidad a seguir durante la redacción del mismo, incluyendo las métricas y las listas de comprobación necesarias. Durante esta fase no pueden acceder a las rúbricas definidas por el equipo docente ya que deben de ser capaces de redactar sus propias rúbricas tomando como referencia los criterios establecidos por la norma UNE 157001. Según lo establecido en su plan de Gestión de la Calidad, van evaluando las partes del proyecto finalizadas, corrigiendo todos aquellos aspectos no conformes con las comprobaciones realizadas. Una vez completado el proyecto y todas las tareas de gestión asociadas, el grupo da por finalizado el proyecto.

El equipo docente evalúa cada proyecto finalizado mediante el módulo de corrección por rúbricas incluido en el sistema de Gestión. Por cada error detectado, el Sistema registra la rúbrica no conforme, con el mensaje y la puntuación correspondiente.

A partir del momento en que se activa la visualización de las correcciones, los alumnos pueden ver y comparar las comprobaciones que han verificado con las rúbricas consideradas no conformes por el equipo docente. Esto permite actuar a los grupos de trabajo en dos sentidos: si encuentran rúbricas consideradas no conformes que coinciden con comprobaciones que han realizado y que han considerado conforme, deben revisar su interpretación de la norma ya que no se corresponde con el objetivo buscado. Por otro lado, si encuentran rúbricas consideradas no conformes sin la comprobación correspondiente dentro de su lista, deben añadirla para verificarla en el siguiente proyecto.

Antes de comenzar el segundo proyecto, los alumnos deben actualizar su plan de Gestión, añadiendo las conclusiones extraídas de las correcciones propuestas por el equipo docente.

Por último y tras la finalización del segundo proyecto, el equipo docente lo corrige y observa si se han reducido o eliminado los errores cometidos en el primer proyecto.
El proceso de trabajo seguido se puede ver en la Figura 1.

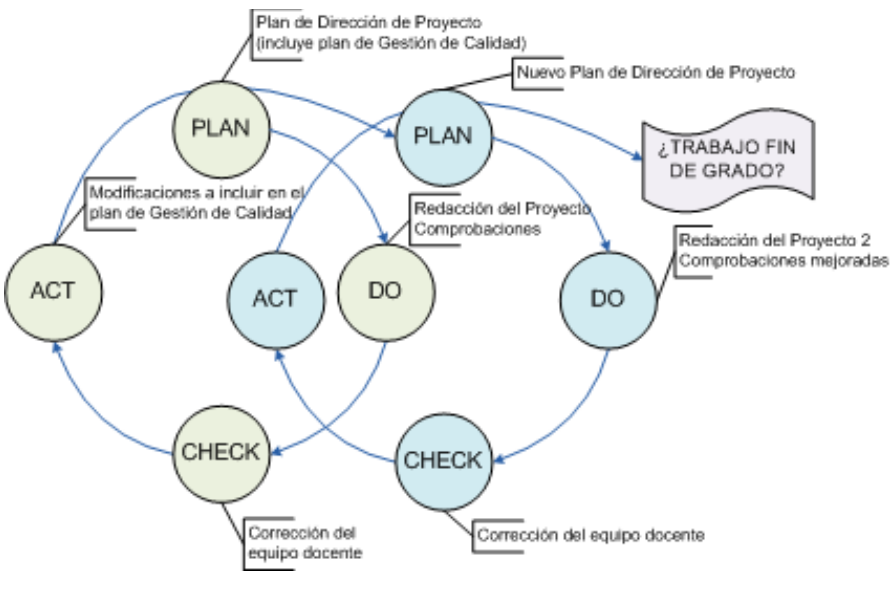

Figura 1. Proceso de trabajo

\section{RESUltados}

Para analizar los resultados de la mejora propuesta, se ha tomado como muestra los trabajos de los 18 grupos que presentaron sus proyectos antes del final del primer semestre.

Un total de 10 grupos de trabajo presentaron su primer proyecto con el tiempo suficiente para que pudiera ser corregido antes de presentar su segundo proyecto. Estos grupos pudieron analizar las rúbricas de las correcciones realizadas por el equipo docente antes de entregar el segundo trabajo. Con esta información tuvieron la oportunidad de mejorar el plan de Gestión de la Calidad, incluyendo las listas de comprobación, para aplicarlo a la redacción del nuevo trabajo. Estos grupos se han identificado con los códigos M01 a M10. Los otros 8 grupos presentaron los dos proyectos de forma simultánea, por lo que aplicaron planes de Gestión de la Calidad similares en los dos proyectos. Estos grupos se han identificado con los códigos N01 a N08. Los grupos se dispondrán en las figuras siguientes ordenados de izquierda a derecha, según la calificación obtenida en la corrección del primer proyecto.

Se analizan primero las calificaciones obtenidas por los grupos en el documento Memoria de sus proyectos. Se centra el análisis en este documento por ser el más extenso (consta de 21 apartados).

La representación gráfica de las calificaciones de los grupos con posibilidad de mejora se pueden observar en Figura 2.

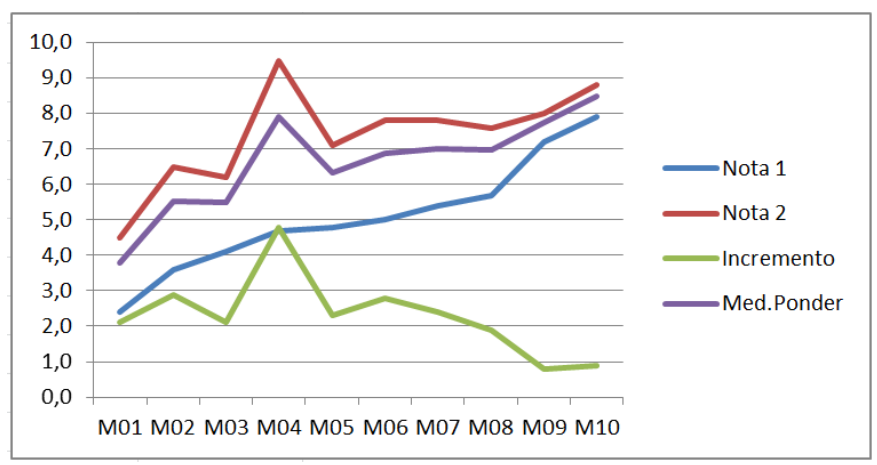

Figura 2. Calificaciones de grupos que pudieron mejorar su plan de Gestión de Calidad 
Siendo Nota 1 la calificación del primer proyecto; Nota 2 la calificación del segundo; Incremento el incremento de la calificación del segundo proyecto respecto del primero; y Med. Ponder. el valor de la media ponderada de las calificaciones, teniendo en cuenta que la calificación del primer proyecto es el $25 \%$ de la calificación final y la del segundo proyecto es el $50 \%$.

Las calificaciones de los grupos que entregaron los proyectos sin tiempo para implementar ninguna mejora en su plan de Gestión de la Calidad se representan en la Figura 3.

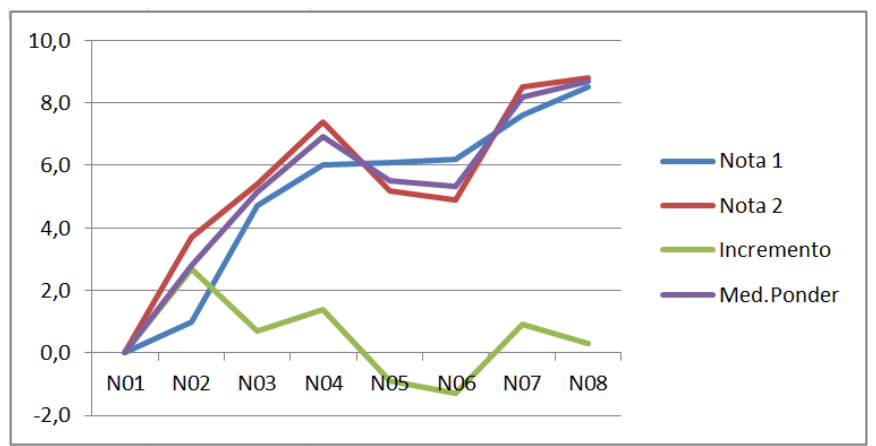

Figura 3. Calificaciones de grupos sin tiempo para implementar ninguna mejora en su plan de Gestión de Calidad

En la Tabla 1 vienen reflejados los valores medios de las calificaciones y de su incremento, así como las desviaciones típicas indicadas entre paréntesis.

Tabla 1 Media y desviación típica de las calificaciones

\begin{tabular}{cccc}
\hline & \multicolumn{2}{c}{ Grupos } & p-valor del \\
\cline { 2 - 3 } & $\mathrm{M}(\mathrm{n}=10)$ & $\mathrm{N}(\mathrm{n}=8)$ & \\
contraste \\
\hline Nota1 & $5.1(1.6)$ & $5.0(3.0)$ & \\
Nota2 & $7.4(1.4)$ & $5.5(2.9)$ & \\
Inc & $2.3(1.1)$ & $0.5(1.3)$ & 0.005 \\
\hline
\end{tabular}

Nota: Nota1 = calificación del primer proyecto; Nota2 = calificación del segundo proyecto; Inc = incremento de la calificación del segundo proyecto respecto del primero; $\mathbf{M}=$ grupos que pudieron mejorar su plan de Gestión de Calidad; $\mathrm{N}$ = grupos sin tiempo para implementar ninguna mejora en su plan de Gestión de Calidad; $\mathrm{n}$ = número de grupos.

Para demostrar si es significativa la diferencia del incremento en las calificaciones entre los grupos que pudieron mejorar su plan de Gestión de Calidad y los que no, se ha hecho un contraste de comparación de dos medias de muestras independientes mediante t de Student cumpliendo normalidad de las muestras e igualdad de varianzas. Para ello se ha utilizado el software estadístico Minitab 17. El resultado ofrece un p-valor de 0.005 (Tabla 1).

Comparando los resultados se pueden extraer las siguientes conclusiones:

- Las dos muestras parten de una calificación media muy similar en el primer proyecto (5.0 y 5.1).

- Todos los grupos que han aplicado la mejora propuesta han aumentado la calificación del segundo proyecto. La mejora media es de 2.3 puntos, con una mejora máxima de 4.8 puntos en el caso del grupo M04.

- Los grupos que no han aplicado mejora han aumentado ligeramente la calificación (0.5 puntos de media) del segundo proyecto, posiblemente por la experiencia adquirida al realizar el primer proyecto. En algunos casos, como en el grupo N05 y N06, la calificación ha disminuido.

- $\quad$ El análisis de contraste efectuado entre las medias de los incrementos en las calificaciones de grupos que pudieron mejorar su plan de Gestión de Calidad y los que no, ofrece un valor de 0.005 , lo cual es una diferencia estadísticamente significativa al nivel del 95 por ciento $(p<0.05)$. La diferencia estimada es de 1.8.

Por último, se analizará el número de apartados con errores en el documento Memoria de los proyectos presentados.

En el caso de los grupos con la oportunidad de mejora, los datos son los ilustrados en la Figura 4.

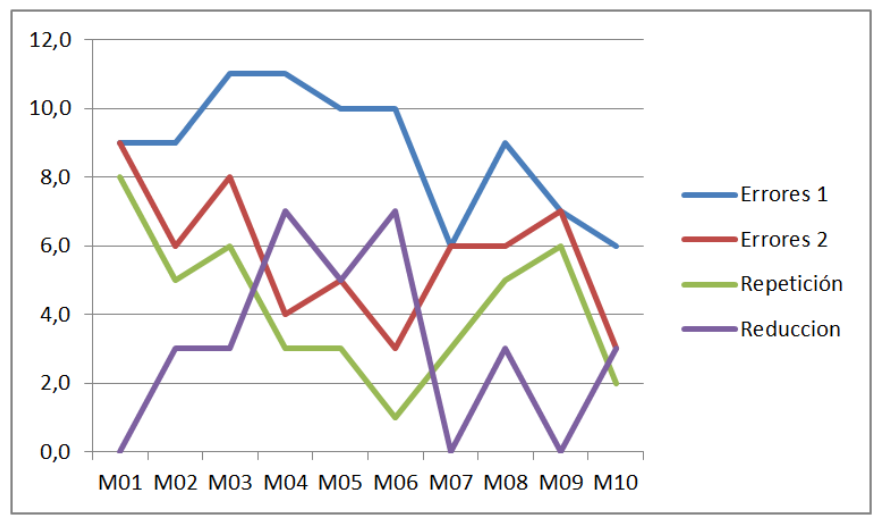

Figura 4. Análisis de los errores de grupos que pudieron mejorar su plan de Gestión de Calidad

Siendo Errores 1 y Errores 2 el número de apartados con errores en los proyectos primero y segundo, respectivamente; Repetición el número de apartados en los que se ha repetido un mismo error en los dos proyectos; y Reducción la diferencia del número de apartados con errores cometidos en el segundo proyecto respecto del primero.

La representación gráfica de los datos correspondientes a los errores cometidos por los grupos que entregaron los proyectos sin posibilidad de mejora, se muestran en la Figura 5.

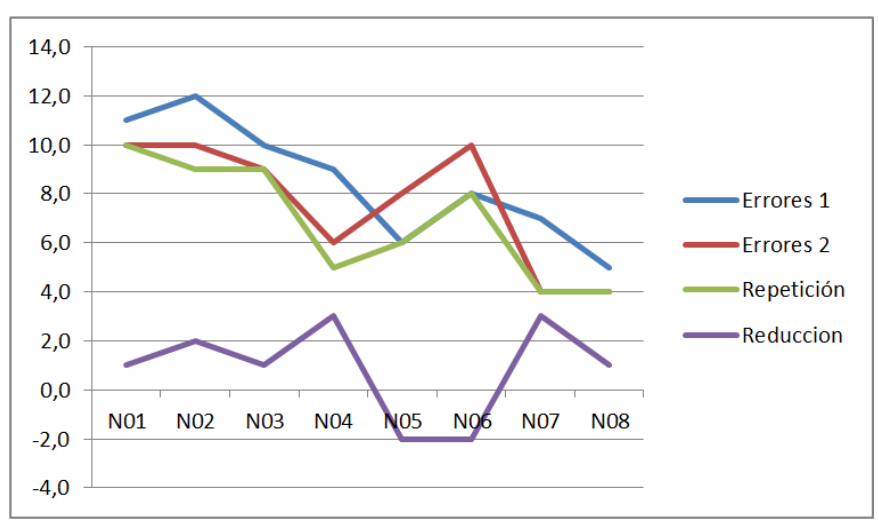

Figura 5. Análisis de los errores de grupos sin tiempo para implementar ninguna mejora en su plan de Gestión de Calidad 
En la Tabla 2 se presentan los valores medios de los errores, de su reducción y de las repeticiones, así como las desviaciones típicas, indicadas entre paréntesis.

Tabla 2 Media y desviación típica de los errores

\begin{tabular}{cccc}
\hline & \multicolumn{2}{c}{ Grupos } & p-valor del \\
\cline { 2 - 3 } & $\mathrm{M}(\mathrm{n}=10)$ & $\mathrm{N}(\mathrm{n}=8)$ & \\
contraste
\end{tabular}

Nota: Errores1 = número de apartados con errores en el primer proyecto; Errores2 = número de apartados con errores en el segundo proyecto; Repet = número de apartados en los que se ha repetido un mismo error en los dos proyectos; Reduc = diferencia del número de apartados con errores cometidos en el segundo proyecto respecto del primero; $\mathrm{M}=$ grupos que pudieron mejorar su plan de Gestión de Calidad; $\mathrm{N}$ = grupos que sin tiempo para implementar ninguna mejora en su plan de Gestión de Calidad; $\mathrm{n}=$ número de grupos.

Para comprobar si son significativas las diferencias del número de apartados con errores repetidos y las diferencias de la reducción de apartados con errores cometidos entre los grupos que pudieron mejorar su plan de Gestión de Calidad y los que no, también se han hecho contrastes de comparación de dos medias de muestras independientes mediante $t$ de Student cumpliendo normalidad de las muestras e igualdad de varianzas. Para ello, de nuevo, se ha utilizado el software estadístico Minitab 17. Los resultados se pueden ver en la Tabla 2.

Comparando los datos de las dos muestras se puede deducir:

- Las dos muestras cometen un número similar de errores en el primer proyecto: 8.8 los grupos que han podido aplicar la mejora y 8.5 errores el resto.

- Los grupos que han podido aplicar la mejora han reducido una media de 3.1 apartados con errores mientras que el resto de grupos no llega a una reducción de un apartado con errores, concretamente una reducción de 0.9 apartados.

- Además, los grupos que han podido aplicar mejora han repetido errores en menor medida que el resto de grupos: 4.2 repeticiones frente a 6.9 .

- El análisis de contraste efectuado entre las medias del número de apartados con errores repetidos de grupos que pudieron mejorar su plan de Gestión de Calidad y los que no, ofrece un valor de 0.024 , luego las medias son significativamente diferentes al nivel del 95 por ciento $(\mathrm{p}<0.05)$. La diferencia estimada es de 2.7.

- El análisis de contraste efectuado entre las medias de la reducción del número de apartados con errores cometidos de grupos que pudieron mejorar su plan de Gestión de Calidad y los que no, ofrece un valor de 0.065. Como el p-valor está muy cerca del 0.05 , sería necesario aumentar el número de las muestras para poder concluir si la reducción del número de apartados con errores entre los grupos es significativa.

\section{CONCLUSIONES}

La experiencia de relacionar las listas de comprobación del plan de Gestión de la Calidad con las rúbricas empleadas en la corrección de los trabajos, ha resultado satisfactoria. Tomando como punto de partida la corrección del primer proyecto, se comprueba que tanto los grupos de trabajo que han podido aplicar la mejora como el resto obtienen una calificación muy similar, en torno a 5 puntos, y cometen el mismo número de errores: 8.8 y 8.5 , respectivamente.

Analizando los resultados de las calificaciones, se ve que existen diferencias significativas entre los grupos que han aplicado la mejora y el resto. Los primeros han aumentado su calificación y los segundos no presentan ninguna mejora. También existe diferencia estadísticamente significativa en el número de apartados con errores repetidos. Los grupos que han podido aplicar mejora han repetido errores en menor medida que el resto de grupos. En cuanto al número de apartados con errores cometidos, aunque se observa una reducción en los grupos que han aplicado las mejoras mientras que en el resto no, el análisis de contraste no es concluyente para decir si las diferencias entre ambos grupos son significativas. Para conseguirlo se necesitaría aumentar el número de grupos en las muestras lo que se logrará al seguir aplicando la experiencia en los próximos cursos.

Se puede comprobar que también se han reforzado las competencias de saber realizar la autoevaluación del trabajo, ya que son los alumnos los que revisan el nivel de calidad de su trabajo respecto a las directrices dadas por la norma, y la competencia de comprender la utilidad de los ciclos de mejora continua, consiguiendo una mejora importante en el segundo proyecto presentado.

Esta experiencia es aplicable a todas las asignaturas de Oficina Técnica, Oficina de Proyectos y Proyectos impartidas en los grados de Ingeniería, ya que en todas se trabajan competencias similares. En principio, también se podría implementar en otras asignaturas, siempre que cumplan con los siguientes aspectos formales: los alumnos deben desarrollar un mínimo de dos trabajos y deben ser capaces de elaborar las listas de comprobación del trabajo realizado, incluidas en un plan de Gestión de Calidad o no. Por último, las correcciones de los trabajos deben realizarse por medio de rúbricas.

Desde el punto de vista de la sostenibilidad de la mejora propuesta no será necesario ninguna inversión de recursos. Una vez desarrollado el módulo de Gestión de Calidad dentro del sistema de Gestión de Oficinas Técnicas y establecido el método de trabajo, no se precisarán más actuaciones excepto las propias del mantenimiento de los sistemas informáticos.

\section{REFERENCIAS}

AENOR. (Junio de 2014). UNE 157001_2014 Criterios generales para la elaboración formal de los documentos que constituyen un proyecto técnico. Madrid: AENOR.

Andrade, H. (2005). Teaching with rubrics. College Teaching, 53(1), 27-30. 
Cano, M. E. (2008). La evaluación por competencias en la educación superior. Recuperado el 8 de mayo de 2017, de https://www.ugr.es/ recfpro/rev123COL1.pdf

Carrizosa, E. (2011). Rúbricas paras la orientación y evaluación del aprendizaje en entornos virtuales. Recuperado el 8 de mayo de 2017, de http://www.uoc.edu/symposia/dret_tic2011/pdf/4.carrizo sa_prieto_esther_gallardo_ballestero_jose.pdf

Cebrián, M., \& Monedero, J. J. (2009). El e-portafolio y la erubrica en la supervisión del practicum. Recuperado el 18 de mayo de 2017, de http://practicum.uma.es/wpcontent/uploads/2011/09/ComMoneCebri.pdf

de Miguel, M. (2005). Modalidades de enseñanza centradas en el desarrollo de competencias. Orientaciones para promover el cambio metodológico en el espacio europeo de educación superior. Recuperado el 8 de mayo de 2017,

https://www2.ulpgc.es/hege/almacen/download/42/4237 6/modalidades_ensenanza_competencias_mario_miguel 2_documento.pdf

Fernandez, A. (2010). La evaluación orientada al aprendizaje en un modelo de formación por competencias en la educación universitaria. Recuperado el 18 de mayo de 2017, de http://redu.net/redu/files/journals/1/articles/144/public/144-130-2PB.pdf

Gatica, F., \& Uribarren, T. (2013). ¿Cómo elaborar una rúbrica? Investigación en Educación Médica, 2(1), 6165.

Goodrich, H. (2000). Using Rubrics to Promote Thinking and Learning. Educational Leadership, 57(5), 13-18.

Granados, G. A. (2013). Evaluación Educativa. Como Proceso de Mejora Continua para la Calidad Educativa. Recuperado el 8 de mayo de 2017, de Visión industrial. Una manera diferente de ver la industria: http://www.visionindustrial.com.mx/industria/en-laeducacion/evaluacion-educativa-como-proceso-demejora-continua-para-la-calidad-educativa

Martínez, J. G. (2008). Las rúbricas en la evaluación escolar: su construcción y su uso. Avances en Medición, 6, 129134.

Menéndez, E. (13 de 02 de 2014). Rúbricas: qué son, cómo se diseñan y herramientas TIC para su elaboración. Recuperado el 8 de mayo de 2017, de Ticteando. Otra forma de trabajar las TIC: http://ticteando.org/rubricasque-son-como-se-disenan-y-herramientas-tic-para-suelbaracion/\#.WSgChOvyjIU

Mertler, C. A. (2001). Designing scoring rubrics for your class-room. Practical Assesment Research and Evaluation. Recuperado el 18 de mayo de 2017, de http://PAREonline.net/getvn.asp?v=7\&n=25

Pickett, N., \& Dodge, B. (2007). Rubrics for Web Lessons. Recuperado el 18 de mayo de 2017, de http://webquest.org/sdsu/rubrics/weblessons.htm

Project Mangement Institute, I. (. (2013). Guía de los Fundamentos para la dirección de proyectos (Guía del PMBOK). Newtown, Pensilvania.

Rodríguez, R. M. (2007). Mejora continua de la práctica docente universitaria: una experiencia desde el proceso de convergencia del Espacio Europeo de Educación Superior. Recuperado el 8 de mayo de 2017, de https://dialnet.unirioja.es/descarga/articulo/2783317.pdf/

Universidad de Zaragoza, E. d. (2016). Estudios de Grado y Máster. Recuperado el 6 de junio de 2017, de Grado en Ingeniería en Diseño Industrual y Desarrollo de Producto:

http://titulaciones.unizar.es/guias16/index.php?asignatur $\mathrm{a}=25821$ 